\title{
Why are diabetics prone to kidney infections?
}

\author{
Michael Zasloff \\ MedStar Georgetown Transplant Institute, Georgetown University School of Medicine, Washington, DC, USA.
}

\begin{abstract}
People with diabetes mellitus are at higher risk of developing serious ascending infections of the urinary tract. The traditional explanation has focused on the role of glycosuria in promoting bacterial growth. Using mouse models, Murtha et al. demonstrate that when the intracellular insulin signaling pathway is compromised, antimicrobial defenses are compromised too, and the mice are unable to effectively handle uropathogenic $E$. coli introduced experimentally into the urinary tract. These observations strongly support the hypothesis that the antimicrobial defenses of the kidney are dependent on insulin, and the urinary tract infections associated with diabetes occur due to reduced expression of these key effectors of innate immunity.
\end{abstract}

\section{Innate immune system} protects against urinary tract infection

Fecal bacteria should have very little difficulty finding their way into the urinary tract considering the reality of our anatomy. So, it is surprising that ascending urinary tract infection (UTI) is not more commonplace. The mechanisms that protect the urinary tract from an ascending bacterial invasion have been uncovered over the past several years. Antimicrobial peptides and proteins appear to play the dominant role. These effectors of the innate immune system are well designed for the task of protecting the urinary tract. They are bactericidal, rapidly acting, broad spectrum, and are expressed as a "cocktail," with each peptide or protein directed at a different bacterial target. This strategy insures that resistance will not develop. When this frontline defense is effective, neutrophils play a minor role and the collateral tissue damage associated with acute inflammation does not occur.

The major components of the urinary tract's antimicrobial armamentarium include defensins $(1,2)$, cathelicidin LL-37 (3), several nonenzymatic ribonucleases
(RNases) (4-6), and lipocalin 2 (Lcn2) (7). The first three act by damaging the bacterial membrane, whereas Lcn2 sequesters the siderophores that bacteria secrete to capture iron needed for growth. The antimicrobial agents are expressed constitutively but can be induced to higher levels should bacteria enter the urinary tract (8). As a bacterial cell approaches the uroepithelium, it faces an increasing concentration of these antimicrobial agents, creating a shield that ultimately prevents a viable microbe from attaching to the surface and expanding in numbers (Figure 1).

The role of these antimicrobial agents in maintaining urine sterility can be demonstrated in a very simple experiment: if a small inoculum of Pseudomonas aeruginosa is introduced into a clean catch sample of human urine, it will not grow unless the RNase7 in that sample is inactivated (for example, through the addition of an inactivating antibody) (4).

One of the most surprising discoveries in this unfolding story involves the intercalated cells (ICs) of the collecting ducts $(9,10)$. These cells, which represent less than $10 \%$ of the cells that comprise the

Related Article: p. 5634

Conflict of interest: The author has declared that no conflict of interest exists.

Reference information: / Clin Invest. 2018;128(12):5213-5215. https://doi.org/10.1172/JCI124922.

collecting ducts, are known to play a role in acidification $(\alpha-\mathrm{IC})$ or alkalization $(\beta-\mathrm{IC})$ of urine. We now know that these cells express and secrete antimicrobial peptides and proteins and represent a major defense against ascending infections of the kidney parenchyma. Adherence of bacteria to the IC stimulates acidification, which also creates a local environment optimal for the functioning of their secreted antimicrobial agents (10).

\section{UTI in diabetics}

Uropathogenic E. coli (UPEC) is the cause of $80 \%$ of UTI. The infection usually begins as a cystitis. UPEC binds to the bladder epithelium, invades the superficial cells, and forms intracellular bacterial communities (11). If the infection is not controlled at this stage, the microbes ascend into the collecting tubules, provoking an intense inflammatory response, recognized clinically as pyelonephritis.

The immune defenses of the urinary tract fail in the setting of diabetes mellitus (DM). A lower UTI in a diabetic has a high likelihood of evolving into pyelonephritis, renal abscess, and necrotizing papillitis (12). Traditionally, glycosuria has been presented as the likely culprit, the glucose providing a more favorable environment for bacterial growth. However, the report in this issue of the JCI by Murtha et al. suggests that urinary sugar is likely not the full story behind UTI in the diabetic (13).

Previously, these investigators had shown that the expression of RNase7 by the uroepithelium could be induced by insulin via the classical insulin signaling pathway (14). Recent studies have shown that the epithelium of the bladder and the ICs of the collecting ducts express a cocktail of potent, broad spectrum antimicrobial peptides and proteins that constrain the growth of UPEC. The expression of several of these antimicrobial proteins was known to be induced by insulin. The report by Murtha et al. in the current issue of the JCI extends this observation to several mouse models of diabetes. UTI was created by 


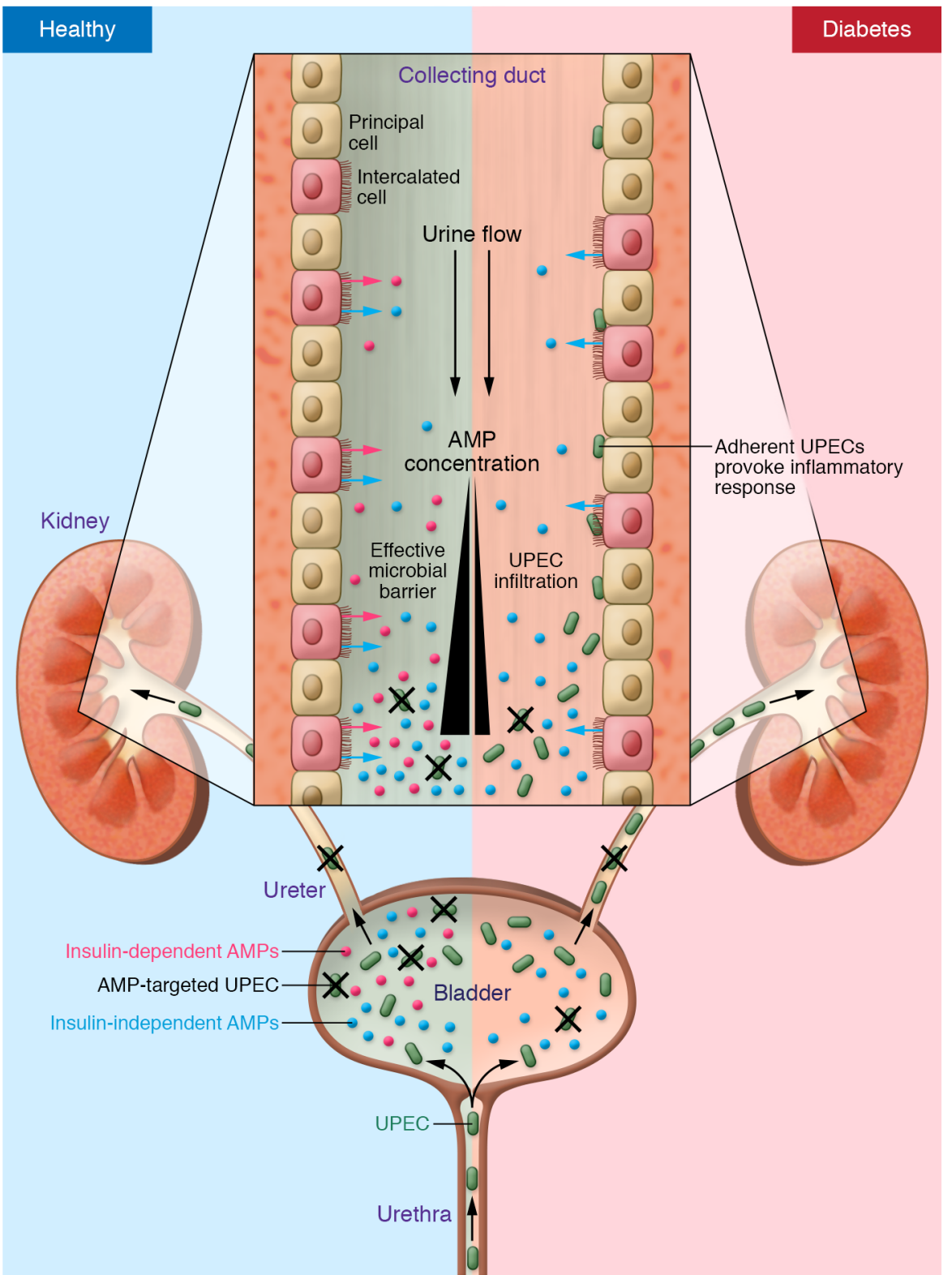

inoculating UPEC transurethrally. The susceptibility of the mice to infection was determined by measuring the concentration of bacteria within the organs at various time points following inoculation.

Several straightforward experiments were first performed. Obese hyperglycemic $d b / d b$ mice, which exhibit a type $2 \mathrm{DM}$ phenotype, demonstrated an increased susceptibility to the UPEC inoculation. To rule out the role of glycosuria, TALLYHO mice, which exhibit reduced insulin sensitivity and impaired glucose tolerance but are not normally hyperglycemic, also failed to control the inoculation of UPEC. Thus, in the mouse, urinary glucose is not the compromising factor.

\section{UTI in mice with the insulin receptor deleted in the ICs}

To more precisely evaluate both the role of the insulin signaling pathway and the IC in renal defense, Murtha et al. constructed a mouse model in which the insulin receptor (IR) was deleted solely from ICs. The animals had normal renal histology and number of ICs, and could properly regulate urine acidification. Transcript and peptide analyses of isolated ICs and whole tissues confirmed that the expression of two key antimicrobial agents, RNase 4 and Lcn2, was significantly depressed in the animals. Samples of urine from the animals failed to suppress the growth of UPEC in contrast to urine from control animals. Most
Figure 1. Increased incidence of UTI in diabetics. In the healthy urinary tract, the epithelium of the urethra, bladder, ureters, and the collecting ducts express and secrete a cocktail of antimicrobial peptides and proteins (AMPs) that effectively constrain fecal uropathogenic E. coli (UPEC) bacteria from ascending into the parenchyma of the kidney. The expression of several of these AMPs, produced by the ICS of the collecting ducts, is dependent on the classical insulin signaling pathway (red arrows). In the direction of the urine flow down the collecting ducts (black arrow), the concentration of the antimicrobial cocktail increases, creating an increasingly more effective immune barrier. In the setting of type 2 diabetes, which is characterized by insulin resistance, expression of the insulin-dependent AMPs is suppressed, creating a less optimal antimicrobial environment, permitting viable microbes to ascend into the collecting ducts. Once attached to the epithelium of the collecting ducts, bacteria invade the epithelial cells, expand in number, and subsequently provoke an acute inflammatory response, clinically recognized as pyelonephritis.

importantly, intraurethral inoculation of UPEC into the IR-IC knockout mice resulted in a greater bladder, kidney, and urine burden than that observed in control animals. Using mouse and human primary renal medullary epithelial cells, Murtha et al. further demonstrated that RNase 4 and Lcn 2 from both sources were induced by the presence of insulin, and that the response could be suppressed by introduction of a PI3K inhibitor (wortmannin) into the system, supporting the role of the classical insulin signaling in induction. As predicted, treatment of wild-type mice with wortmannin reduced their defense against a transurethral inoculation of UPEC compared with untreated animals (Figure 1). 
Extending the story to humans, Murtha et al. measured the urine concentrations of RNase 4 and Lcn 2 in samples from children and adolescents who were healthy and from those with type 2 diabetes. Those with diabetes had lower mean concentrations of the two antimicrobial proteins, which correlated negatively with hemoglobin A1c levels.

\section{Future directions}

Murtha et al. have provided us with a deeper insight into the etiology of UTI that occurs in the setting of diabetes. What can we do with this information, above and beyond what is now the standard of care for diabetics? One could imagine developing a drug that increased insulin sensitivity within the kidney, such as a compound that inhibited PTP1b, the phosphatase that shuts off the activated insulin receptor. Another approach could exploit the discovery that the expression of several of the antimicrobial peptides produced by mammals are induced by simple organic compounds, such as sodium butyrate (15), isoleucine (16), vitamin D3 (17), and estrogen (18). Perhaps similar compounds can be found that could safely boost the expression of renal RNase 4 and Lcn2. Hopefully, the excit- ing story presented by Murtha et al. will stimulate further studies on the innate immunity of the urinary tract.

Address correspondence to: Michael Zasloff, Georgetown University Hospital 2 PWC, 3800 Reservoir Road NW, Washington, DC 20007, USA. Phone: 484.433.7807; Email: maz5@georgetown.edu.

1. Ganz T. Defensins in the urinary tract and other tissues. J Infect Dis. 2001;183(suppl 1):S41-S42.

2. Becknell B, et al. Expression and antimicrobial function of beta-defensin 1 in the lower urinary tract. PLoS One. 2013;8(10):e77714.

3. Chromek M, et al. The antimicrobial peptide cathelicidin protects the urinary tract against invasive bacterial infection. Nat Med.2006;12(6):636-641.

4. Spencer JD, et al. Ribonuclease 7 is a potent antimicrobial peptide within the human urinary tract. Kidney Int. 2011;80(2):174-180.

5. Spencer JD, et al. Ribonuclease 7, an antimicrobial peptide upregulated during infection, contributes to microbial defense of the human urinary tract. Kidney Int. 2013;83(4):615-625.

6. Wang H, Schwaderer AL, Kline J, Spencer JD, Kline D, Hains DS. Contribution of structural domains to the activity of ribonuclease 7 against uropathogenic bacteria. Antimicrob Agents Chemother. 2013;57(2):766-774.

7. Forster CS, et al. Urinary NGAL deficiency in recurrent urinary tract infections. Pediatr Nephrol. 2017;32(6):1077-1080.

8. Becknell B, Schwaderer A, Hains DS, Spencer JD. Amplifying renal immunity: the role of anti- microbial peptides in pyelonephritis. Nat Rev Nephrol. 2015;11(11):642-655.

9. Chassin C, Tourneur E, Bens M, Vandewalle A. A role for collecting duct epithelial cells in renal antibacterial defences. Cell Microbiol. 2011;13(8):1107-1113.

10. Paragas N, et al. $\alpha$-Intercalated cells defend the urinary system from bacterial infection. J Clin Invest. 2014;124(7):2963-2976.

11. Spaulding CN, Hultgren SJ. Adhesive pili in UTI pathogenesis and drug development. Pathogens. 2016;5(1):E30

12. Nitzan O, Elias M, Chazan B, Saliba W. Urinary tract infections in patients with type 2 diabetes mellitus: review of prevalence, diagnosis, and management. Diabetes Metab Syndr Obes. 2015;8:129-136.

13. Murtha MJ, et al. Insulin receptor signaling regulates renal collecting duct and intercalated cell antibacterial defenses. J Clin Invest. 2018;128(12):5634-5646.

14. Eichler TE, et al. Insulin and the phosphatidylinositol 3-kinase signaling pathway regulate Ribonuclease 7 expression in the human urinary tract. Kidney Int. 2016;90(3):568-579.

15. Schauber J, et al. Expression of the cathelicidin LL-37 is modulated by short chain fatty acids in colonocytes: relevance of signalling pathways. Gut. 2003;52(5):735-741.

16. Fehlbaum P, Rao M, Zasloff M, Anderson GM. An essential amino acid induces epithelial $\beta$-defensin expression. Proc Natl Acad Sci U S A. 2000;97(23):12723-12728.

17. Gombart AF. The vitamin D-antimicrobial peptide pathway and its role in protection against infection. Future Microbiol. 2009;4(9):1151-1165.

18. Luthje $P$, et al. Estrogen supports urothelial defense mechanisms. Sci Transl Med. 2013;5(190):190ra80. 